\title{
In the news
}

\section{SYNTHETIC CHROMOSOME}

Synthetic biology approaches have enabled the synthesis of whole bacterial genomes. A team led by Jef Boeke (New York University, USA) and Srinivasan Chandrasegaran (Johns Hopkins University, USA) now describe, in the journal Science, the redesign and construction of an entire eukaryotic chromosome. They termed it Synlll, to designate it is a synthetic version of Saccharomyces cerevisiae chromosome III. Paul Freemont of Imperial College, UK, who was not involved in the study, said that this "provides a proof of concept that complicated chromosomes can be redesigned, synthesised and made to work in a living cell" (The Independent, 28 Mar 2014).

The chromosome III sequence was first redesigned to include desired mutations. The construction comprised an assembly of DNA blocks of increasing size, which was followed by the replacement of the native chromosome by homologous recombination in yeast cells. The resulting Synlll measures only 272,871 bp compared to the 316,617 bp of chromosome III. Describing the work, Dr Boeke said that "What's really exciting about it is the extent to which we have changed the sequence and still come out with a happy healthy yeast at the end [and] we have taught it a few tricks by inserting some special widgets into its chromosome." (BBC News, 27 Mar 2014).

The Synlll mutations include deletions of genetic elements, such as transposons and introns, as well as the flanking of non-essential genes by Cre-recombinase recognition sites to enable genome scrambling. George Church of Harvard University, USA, also commended the work but added that "[the] impacts — good and bad - typically do not become significant until these strategies are applied genome-wide, and this paper represents roughly 2.5 percent of the yeast genome." (The Scientist, 27 Mar 2014). 